\title{
Application of sampled-data control by using vibration manipulation function to suppress residual vibration of travelling crane
}

\author{
Shigeo KOTAKE*, Kazunori YAGI* and Tatsuya TAKIGAMI* \\ *Department of Mechanical Engineering, Mie University, \\ 1577 Kurimamachiya-cho, Tsu city, Mie, 514-8507, Japan \\ E-mail: kotake@mach.mie-u.ac.jp
}

Received 9 January 2015

\begin{abstract}
Vibration manipulation function (VMF) is applied as a linear-piecewise feedforward function to suppress residual vibration of a hoisting load in a one-dimensional overhead travelling crane. The system is modeled as a pendulum of a one-degree-of-freedom (1DOF) oscillator with enforced acceleration of a trolley. When an initial swing angle of the wire of the crane is small, residual vibration of the load can be vanished by this method in one natural period of the oscillator. On the contrary, in case of a large initial angle, certain amount of residual vibration remains because of the nonlinearity of the pendulum. However, it can be eliminated with repeated enforce accelerations of the trolley under VMF in every natural period. Moreover, in case of the existence of external noises and errors, the intermittent repeated accelerations can remove residual vibration with sampleddata feedback control in every natural period. Some examples of numerical simulations and experimental results are shown to validate the abilities of VMF in the crane operation. Residual vibration of the load can be suppressed in any time of the crane work, as far as the trolley travels in constant speed including static position, i.e. in any inertial frame, at the beginning of each operation. This feedforward function could be used for an automatic crane machine with or without the sampled-data feedback control. Since the function is an analytic solution of an intrinsic repowering operation, it can also be used in tutoring software for operator's training.
\end{abstract}

Key words : Crane, Feedforward control, Vibration control, Sampled-data control, Non-linear oscillation

\section{Introduction}

Suppression of residual vibration of an overhead travelling crane has been studied by many researchers from the requirements of industries to reduce accidents during moving a hoisting load and to shorten its operational time with increasing efficiency. Since we hardly use dampers in the system, operations of a trolley of the carne is one of few ways to reduce the vibrations. Although well-trained operators of cranes intrinsically know certain repowering procedures of the trolley to decrease residual vibrations of the load effectively, an analytical function of the repowering operation has not been clarified to be used in an automation system.

Most of the former studies have tried to decide a trajectory of the trolley to reduce the vibrations with an open-loop approach. Auernig and Troger (1987) have decided the trajectory of the trolley from an extension of the Pontryagin's maximum principle. Manson (1982) has studied time-optimal open-loop control. Some studies have treated the trajectory as a linear combination of certain basis functions, which can be optimized with heuristic trials such as genetic algorithms (Kojima and Habiro, 2003). Other studies have used a similar linear combination of functions to optimize trajectories by minimizing a certain evaluation function such as an integral of jerks (Hindle and Singh, 2001). However, since the openloop control is sensitive to system control parameters, external noises and control errors can decrease the robustness of the system. Moreover, the former suppression methods used rather artificial feedforward functions, which have not precisely been formulated under the background of analytical dynamics.

Other researchers shape arbitral input functions by using input/command shaping techniques (Singhose, 2003). In 
this method, vibration created at the first half will be canceled out by putting inverse signal at the second half. Although this method is widely used for more than 50 years, there still exist several weak points in this technique. At first, it only works for suppression of vibration, but not for excitation. Thus it does not provide arbitral manipulating operation for a 1DOF oscillator. In second, this method is not robust from external noise, since it cannot form closed loop with feedback signals. Although some attempts have been reported (Chang, et al., 2001), they have not succeeded so much.

Other researchers have sought feedback controls which are less sensitive from parameter variations and disturbances. They varies from conventional PID to intelligent approaches. Since feedback sensors increase cost and complication of the system, some studies try to reduce the number of sensors in the closed-loop control (Solihin and Wahyudi, 2007). Moreover, online trajectory generations have been performed under feedback loop with PD controller (Sun and Fang, 2014). However, perfect suppressions of residual vibration in the overhead travelling crane have not been achieved within a natural period of the pendulum oscillation by using these methods.

Since proper trajectory of the trolley had hardly been obtained from former studies, recent researchers have applied fuzzy adaptation rule to generate speed patterns of the load in the closed-loop system (Yubazaki and Hirota, 2003). Although a trained fuzzy neural network can control the crane well, they sometimes fail to suppress residual vibrations and no analytical formulations of the trajectory cannot be deduced from these methods. As Kang and Bien (2000) pointed out, controlling a crane system is a multi-objective satisfactory problem; one is positioning of the trolley at an aimed point and the other is anti-swing of the hoisting load as possible. Apparently, since two objectives contradict each other, they make the solution difficult to be found.

Recently, we have found an analytical feedforward function, called vibration manipulation function (VMF), to control one mass-spring system (Kotake, et al., 2013, Kotake, et al., 2014). By determining enforced displacement trajectory of the spring base of the oscillator from the functions, the spring mass can be brought to the aimed position and velocity in its natural period. Hence the position and velocity of the base can be independent from those of the mass under discrete dynamics in every natural period (Takata, et al., 2011), the above multi-objective satisfactory problem has been avoided.

Although perfect suppression of residual vibration in a linear oscillator of one-degree-of-freedom (1DOF) can be achieved by using the analytic function theoretically, it requires certain robustness from external noises or misprediction of observations in sampling. Moreover, since the travelling crane is a pendulum oscillator, it demands certain idea to compensate the function to control the nonlinear system. However, since VMF can be modified to reduce the difference between aimed and actual quantities at every natural period, robust sampled-data feedback control can be realized (Kotake, et al., 2014). In the system, errors or noises or nonlinearities can be compensated by repeated modifications in discrete region, which solve the problem of infinite-dimensional model in lifting techniques under sampled-data control (Yamamoto, 1994). Moreover, since input function can be generated from the series of discrete aimed values, which can either suppress or excite the oscillation.

In this study, we have developed a method to suppress residual vibration of the hoisting load during the operation of the overhead travelling crane by using VMF. We have numerically calculated the time evolution of the position of the load, $x(t)$, in the one-dimensional crane with enforced displacement of the trolley, $X_{t r}(t)$, determined from the analytical function of VMF. Moreover, we demonstrated suppression of residual vibration of the hoisting load in experiments. And comparing our method with the well-known input/command shaping approach, we clarify the advantages and disadvantages of the proposed method. Since VMF is deduced from the motion of a virtual three-vibro-impact oscillator, some parameters in the function are defined from the state of the three oscillators. In this study, we will show how to apply VMF to suppress residual vibration of the overhead travelling crane in an inertial flame. In the following discussion, hence the sampled-data control method for a linear oscillator of 1DOF has already been discussed in former study (Kotake, et al., 2014), we will refer it as "the control of a linear oscillator" in this paper.

\section{Model of a one-dimensional overhead travelling crane with a trolley}

As shown in Fig. 1, the one-dimensional overhead travelling crane consists of a trolley, a wire and a hoisting load. The load is regarded as a material particle with a mass of $m$. The wire is considered as a flexible rod with a length of $l$, of which mass is ignored compared with the load mass. Having a mass of $M$, the trolley moves on a horizontal strait rail. Assumed no damping exists in the system, the overhead travelling crane can be treated as a pendulum, of which base can be moved arbitrary with trolley by applied force $F$. The one-dimensional dynamical equations of the overhead travelling 
crane are given by

$$
\begin{aligned}
& \ddot{X}_{t r} \cos \theta+l \ddot{\theta}+g \sin \theta=0 \\
& (m+M) \ddot{X}_{t r}+m l \ddot{\theta} \cos \theta-m l \dot{\theta}^{2} \sin \theta=F
\end{aligned}
$$

from the Lagrange's equation of motion (Sun and Fang, 2014). Here, variables $X_{t r}, \theta, F$ denote trolley position, the wire swing angle, and the applied force to the trolley, respectively. And $g$ is the gravity acceleration. The position of the trolley is positive, when it locates at the right side of the rail origin. The swing angle of the wire is positive if it rotates counterclockwise from pendent position. The driving force toward right direction is positive.

In this overhead travelling crane, we try to bring the hoisting load from static initial position to static destination in short time without residual vibration. Under this condition, three state variables, trolley velocity $X_{t r}$, swing angle of the wire, $\theta$, and swing angular velocity of the wire, $\dot{\theta}$, are required to converge to zero at the beginning and at the end of the control. Moreover, since residual vibration should be eliminated under constant speed of travelling, the wire swing angular velocity, $\dot{\theta}$, is required to converge to zero.

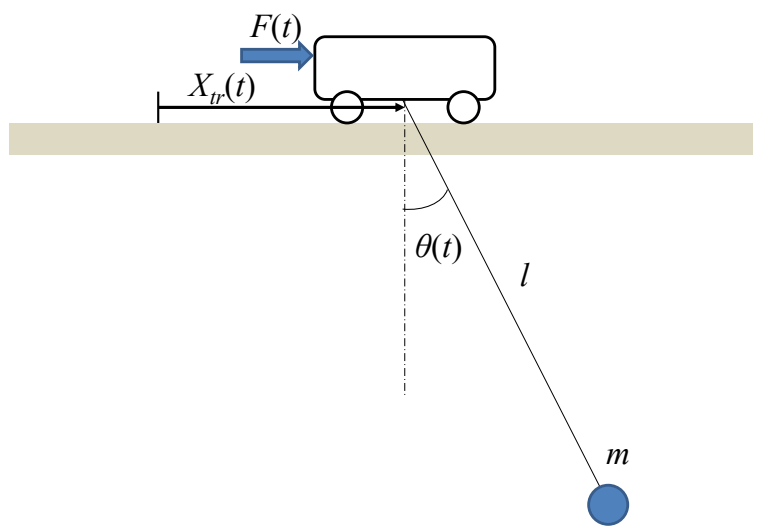

Fig. 1 Schematic figure of the one-dimensional overhead travelling crane, which consists of a trolley, a wire and a hoisting load

\section{Trajectory of trolley to manipulate overhead travelling crane}

\subsection{Linearized dynamic equations of overhead travelling crane}

To suppress residual vibration of the hoisting load in the overhead travelling crane, we have to manipulate the trolley under Eq. (1) and (2). To focus on the properties of VMF, we merely discuss the Eq. (1) at first. Hence Eq. (2) will be used to determine the way to move the trolley in practice, it will be discussed shortly in afterward.

In case of small swing angle of the wire, $\theta<<1$, weak nonlinear differential equation of Eq. (1) can be approximated into the following linear differential equation.

$$
\ddot{X}_{t r}+l \ddot{\theta}+g \theta=0
$$

By rewriting the equation, the following formula will be obtained,

$$
\ddot{\theta}=-g / l\left(\theta+\ddot{X}_{t r} / g\right)=-\omega_{\mathrm{t}}^{2}\left(\theta+\ddot{X}_{t r} / g\right)
$$

where $\omega_{t}=\sqrt{g / l}$ is the natural angular frequency of the swing angle of the wire in the overhead travelling crane and the natural period of the crane is defined as $\tau=2 \pi / \omega_{\mathrm{t}}$. In the following we use non-dimensional units. Characteristic time is $1 / \omega_{\mathrm{t}}$, characteristic acceleration is $g$ and characteristic mass is unit mass. Therefore characteristic length is also $g$. Under the non-dimensional units, the one-dimensional dynamical equation of the overhead travelling crane becomes in the following, 


$$
\ddot{\theta}=-\theta-\ddot{X}_{t r}
$$

\subsection{Trajectory of trolley obtained from vibration manipulation function}

By regarding the non-dimensional acceleration of the trolley as a negative virtual enforced base angle, $\Theta(t)$,

$$
\ddot{X}_{t r}(t)=-\Theta(t)
$$

Therefore, in the oscillation of the overhead travelling crane, the derivative equation of the swing angle, $\theta$, becomes as following equation of a $1 \mathrm{DOF}$ rotational oscillator:

$$
\ddot{\theta}=-\theta+\Theta
$$

According to the control of a linear oscillator (Kotake, et al., 2014), a mass of a linear oscillator of 1DOF can be controlled in one natural period by VMF as enforced displacement of a base of the oscillator. As shown in Eq. (7), since the dynamical equation of the 1DOF rotational oscillator is equivalent to the dynamical equation of the 1DOF oscillator, the swing angle can be controlled by determining the virtual enforced base angle, $\Theta(t)$, along VMF in the rotational oscillator of the pendulum. When the enforced base angle operates from starting time of $t_{0}$ during the natural period of $\tau$ as an operational period. Time during operational period can be expressed as $t=t_{0}+t^{\prime}$, where $0<t^{\prime}<\tau$.

To control the load from the initial swing angle of $\theta$ in $=\theta\left(t_{0}\right)$ and the initial swing angular velocity of $\dot{\theta}_{\text {in }}=\dot{\theta}\left(t_{0}\right)$ to the aimed swing angle of $\theta \mathrm{en}=\theta\left(t_{0}+\tau\right)$ and the aimed swing angular velocity of $\dot{\theta}_{\mathrm{en}}=\dot{\theta}\left(t_{0}+\tau\right)$, the virtual enforced base angle of the pendulum from its pendent position should be in the following equation:

$$
\begin{aligned}
\Theta\left(t_{0}+t^{\prime}\right)=\sum_{p} & \alpha_{p}\left[-\left\{2 \Theta\left(t_{0}\right)+\left(\omega_{+}^{2}-1\right)\left(\theta_{i n}-\theta_{e n}\right)\right\}\left(1-\omega_{-}^{2}\right) \omega_{+} \omega_{-} \cos \left(\omega_{-} t^{\prime}\right)\right. \\
& +\left\{2 \Theta\left(t_{0}\right)+\left(\omega_{-}^{2}-1\right)\left(\theta_{i n}-\theta_{e n}\right)\right\}\left(1-\omega_{+}^{2}\right) \omega_{+} \omega_{-} \cos \left(\omega_{+} t^{\prime}\right) \\
& -\left\{2 \dot{\Theta}\left(t_{0}\right)+\left(\omega_{+}^{2}-1\right)\left(\dot{\theta}_{i n}-\dot{\theta}_{e n}\right)\right\}\left(1-\omega_{-}^{2}\right) \omega_{+} \sin \left(\omega_{-} t^{\prime}\right) \\
& \left.+\left\{2 \dot{\Theta}\left(t_{0}\right)+\left(\omega_{-}^{2}-1\right)\left(\dot{\theta}_{i n}-\dot{\theta}_{e n}\right)\right\}\left(1-\omega_{+}^{2}\right) \omega_{-} \sin \left(\omega_{+} t^{\prime}\right)\right] / 2 \omega_{+} \omega_{-}\left(\omega_{-}^{2}-\omega_{+}^{2}\right) \\
& =\sum_{p} \alpha_{p} \Theta_{p}\left(t_{0}+t^{\prime}\right)
\end{aligned}
$$

where $\omega_{+}=p+1 / 2$ and $\omega_{=}=1 / 2$ with natural number of $p$. These are two angular eigen-frequencies in a coupled rotational oscillator of a large rotational oscillator and the mass center of two small rotational oscillators in three-vibro-impact rotational oscillator as an analogy of the three-vibro-impact linear oscillator (Kotake, et al., 2014). In the VMF of Eq. (8), parameter $\alpha_{p}$ stands in the following equation:

$$
\sum_{p} \alpha_{p}=1
$$

where some $\alpha_{\mathrm{p}}$ are non-zero and the others are zero. We can determine these parameters to optimize the trajectory of the load during the operation. Hence large number of p requires large acceleration of the trolley, $\alpha_{1}$ should be 1 and the others, $\alpha_{p \neq 1}$, should be 0 in case of the overhead travelling crane. Moreover, in the function of Eq. (8), the following relations always stand;

$$
\Theta\left(t_{0}\right)=-\Theta\left(t_{0}+\tau\right), \quad \dot{\Theta}\left(t_{0}\right)=-\dot{\Theta}\left(t_{0}+\tau\right)
$$

As shown in Eq. (6), acceleration of the trolley has the relation with the virtual enforced base angle of the pendulum. By double time integral of Eq. (6), we obtain the trajectory of the trolley to manipulate the swing angle of the overhead travelling crane in the following: 


$$
\begin{aligned}
X_{t r}\left(t_{0}+t^{\prime}\right)=\sum_{p} & -\alpha_{p} g\left[\left\{2 \Theta\left(t_{0}\right)+\left(\omega_{+}^{2}-1\right)\left(\theta_{\text {in }}-\theta_{e n}\right)\right\}\left(1-\omega_{-}^{2}\right)\left(\omega_{+} / \omega_{-}\right) \cos \left(\omega_{-} t^{\prime}\right)\right. \\
& -\left\{2 \Theta\left(t_{0}\right)+\left(\omega_{-}^{2}-1\right)\left(\theta_{i n}-\theta_{e n}\right)\right\}\left(1-\omega_{+}^{2}\right)\left(\omega_{-} / \omega_{+}\right) \cos \left(\omega_{+} t^{\prime}\right) \\
& +\left\{2 \dot{\Theta}\left(t_{0}\right)+\left(\omega_{+}^{2}-1\right)\left(\dot{\theta}_{i n}-\dot{\theta}_{e n}\right)\right\}\left(1-\omega_{-}^{2}\right)\left(\omega_{+} / \omega_{-}^{2}\right) \sin \left(\omega_{-} t^{\prime}\right) \\
& \left.-\left\{2 \dot{\Theta}\left(t_{0}\right)+\left(\omega_{-}^{2}-1\right)\left(\dot{\theta}_{\text {in }}-\dot{\theta}_{e n}\right)\right\}\left(1-\omega_{+}^{2}\right)\left(\omega_{-} / \omega_{+}^{2}\right) \sin \left(\omega_{+} t^{\prime}\right)\right] / 2 \omega_{+} \omega_{-}\left(\omega_{-}^{2}-\omega_{+}^{2}\right)+\dot{X}\left(t_{0}\right) t^{\prime} \\
& +X\left(t_{0}\right)=\sum_{p} \alpha_{p} X_{t r}\left(t_{0}+t^{\prime}\right)+\dot{X}_{t r}\left(t_{0}\right) t^{\prime}+X_{t r}\left(t_{0}\right)
\end{aligned}
$$

By solving the differential equation of Eq. (4), we also obtain the trajectory of the swing angle of the wire in the following:

$$
\begin{aligned}
\theta\left(t_{0}+t^{\prime}\right)=\sum_{\mathrm{p}} & \alpha_{\mathrm{p}}\left[\left(\omega_{-}^{2}-\omega_{+}^{2}\right)\left\{\left(\theta_{\mathrm{in}}+\theta_{\mathrm{en}}\right) \cos \left(t^{\prime}\right)+\left(\dot{\theta}_{\mathrm{in}}+\dot{\theta}_{\mathrm{en}}\right) \sin \left(t^{\prime}\right)\right\}\right. \\
& -\left\{2 \Theta\left(t_{0}\right)-\left(1-\omega_{+}^{2}\right)\left(\theta_{\mathrm{in}}-\theta_{\mathrm{en}}\right)\right\} \cos \left(\omega_{-} t^{\prime}\right)+\left\{2 \Theta\left(t_{0}\right)-\left(1-\omega_{-}^{2}\right)\left(\theta_{\mathrm{in}}-\theta_{\mathrm{en}}\right)\right\} \cos \left(\omega_{+} t^{\prime}\right) \\
& -\left\{2 \dot{\Theta}\left(t_{0}\right)-\left(1-\omega_{+}^{2}\right)\left(\dot{\theta}_{\mathrm{in}}-\dot{\theta}_{\mathrm{en}}\right)\right\} \sin \left(\omega_{-} t^{\prime}\right) / \omega_{-} \\
& \left.+\left\{2 \dot{\Theta}\left(t_{0}\right)-\left(1-\omega_{-}^{2}\right)\left(\dot{\theta}_{\mathrm{in}}-\dot{\theta}_{\mathrm{en}}\right)\right\} \sin \left(\omega_{+} t^{\prime}\right) / \omega_{+}\right] /\left\{2\left(\omega_{-}^{2}-\omega_{+}^{2}\right)\right\}=\sum_{p} \alpha_{p} \theta_{p}\left(t_{0}+t^{\prime}\right)
\end{aligned}
$$

\section{Feedforward control to suppress residual vibration during travelling in inertial flame}

\subsection{In case of small initial swing angle}

In this section, we will show some examples of feedforward control of the overhead travelling crane for the suppression of residual vibration by using VMF, when the trolley travels under constant speed in inertial flame before the beginning and after the end of the operation. Suppose at the beginning of the operation, the swing angle is maximized, $\theta\left(t_{0}\right)=\theta$ in, and the swing angular velocity is zero, $\dot{\theta}\left(t_{0}\right)=\dot{\theta}_{\text {in }}=0$. At the end the operation of residual vibration suppression, both of the swing angle and the swing angular velocity are zero; $\theta\left(t_{0}+\tau\right)=\theta$ en $=0$ and $\dot{\theta}\left(t_{0}+\tau\right)=\dot{\theta}$ en $=0$. Since the velocity of the trolley is constant at the start, $\dot{X}_{t r}\left(t_{0}\right)=\dot{X}_{t r}$,in, the acceleration and the jerk of the trolley are zero, $\ddot{X}_{t r}\left(t_{0}\right)=0$ and $\dddot{X}_{t r}\left(t_{0}\right)=0$. Moreover, since the velocity of the trolley is constant at the end, $\dot{X}_{t r}\left(t_{0}+\tau\right)=\dot{X}_{t r \text {,en, the }}$ acceleration and the jerk of the trolley are also zero, $\ddot{X}_{t r}\left(t_{0}+\tau\right)=0$ and $\dddot{X}_{t r}\left(t_{0}+\tau\right)=0$. We place the origin of the coordinate at the beginning position of the operation; $X_{t r}\left(t_{0}\right)=0$.

Hence $\Theta\left(t_{0}\right)=\Theta\left(t_{0}+\tau\right)=0$ and $\Theta\left(t_{0}\right)=\dot{\Theta}\left(t_{0}+\tau\right)=0$ from Eq. (8), from Eq. (11) the trajectory of the trolley should follow the following equation:

$$
\begin{aligned}
X_{t r}\left(t_{0}+t^{\prime}\right)=\sum_{p} & -\alpha_{p} g\left[\left(\omega_{+}^{2}-1\right) \theta_{i n}\left(1-\omega_{-}^{2}\right)\left(\omega_{+} / \omega_{-}\right) \cos \left(\omega_{-} t^{\prime}\right)\right. \\
& -\left(\omega_{-}^{2}-1\right) \theta_{i n}\left(1-\omega_{+}^{2}\right)\left(\omega_{-} / \omega_{+}\right) \cos \left(\omega_{+} t^{\prime}\right) / 2 \omega_{+} \omega_{-}\left(\omega_{-}^{2}-\omega_{+}^{2}\right)+\dot{X}_{i n} t^{\prime} \\
& =\sum_{p} \alpha_{p} X_{t r}\left(t_{0}+t^{\prime}\right)+\dot{X}_{t r_{i n}} t^{\prime}
\end{aligned}
$$

By using Eq. (13) as a trajectory of the trolley, we had simulated the motion of the overhead travelling crane. In the following calculations, we used $\alpha_{\mathrm{p}}=1$ and $\alpha_{\mathrm{p} \neq 1}=0$ as the parameters. The crane was accelerated to suppress residual vibration of the hoisting load during $t_{0}=2 \pi<t^{\prime}<3 \pi$ intermittently during travelling. The acceleration started at the maximum swing angle of $\theta$ in $=0.1$ rad. Fig. 2 (a) shows temporal change of the positions of the trolley and the hoisting load. Fig. 2 (b) shows temporal change of the velocity of the trolley and the swing angular velocity. Here $x$ is the position of the load expressed in the following equation:

$$
x\left(t_{0}+t^{\prime}\right)=l \theta\left(t_{0}+t^{\prime}\right)+X_{t r}\left(t_{0}+t^{\prime}\right)
$$


From the results of numerical calculations, we found fairly well suppression of residual vibration can be realized in one natural period, in case of a small initial swing angle of $\theta$ in $=0.1 \mathrm{rad}$. Since it stands in inertial flame, proposed method can be applicable not only for constant travelling but also for static state of the crane. During the suppression operation, the position of the trolley will be translated from the original position according to Eq. (13).

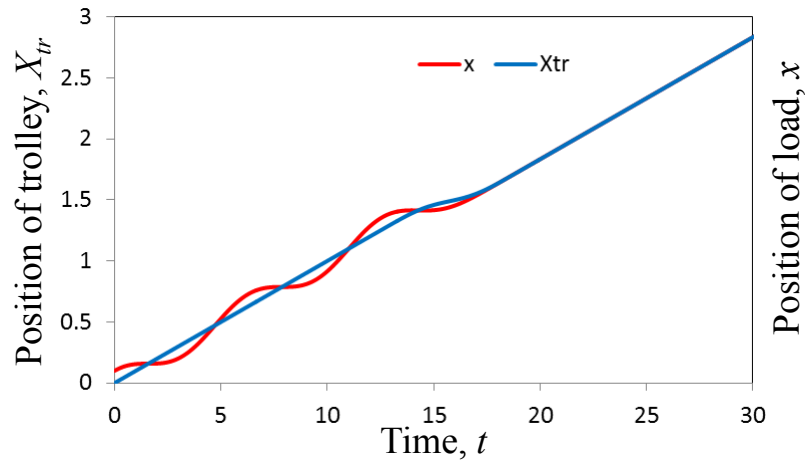

(a)

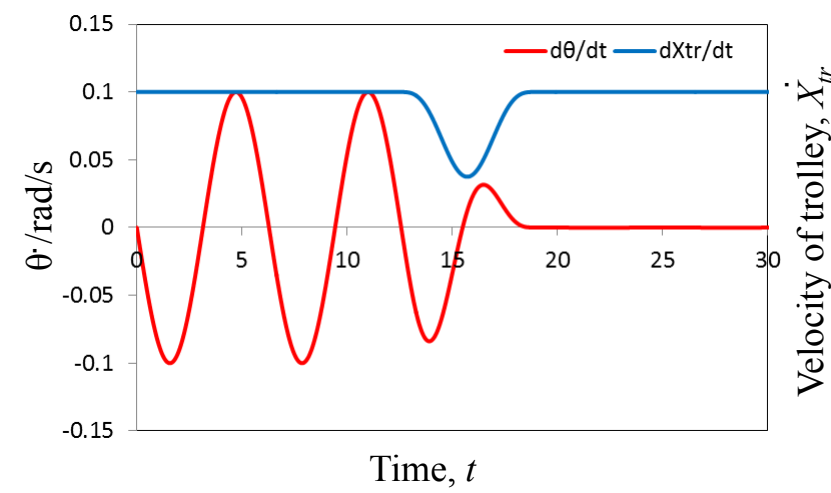

(b)

Fig. 2 Temporal change of (a) the positions of the trolley and the hoisting load or (b) the swing angular velocity and the velocity of the trolley of the crane under the numerical calculation of the suppression of residual vibration with once intermittent enforced displacement during one natural period, in case the initial angle is $0.1 \mathrm{rad}$

\subsection{In case of large initial swing angle}

In case of a large initial angle of the crane, $\theta$ in $=1.0 \mathrm{rad}$ as the maximum angle, Fig. 3 (a) shows temporal change of the positions of the trolley and the hoisting load. Fig. 3 (b) shows temporal change of the velocity of the trolley and the swing angular velocity. Different from the previous results, certain amount of residual vibration remained after one feedforward operation, which indicates residual vibration of the overhead travelling crane cannot be suppressed in one natural period.

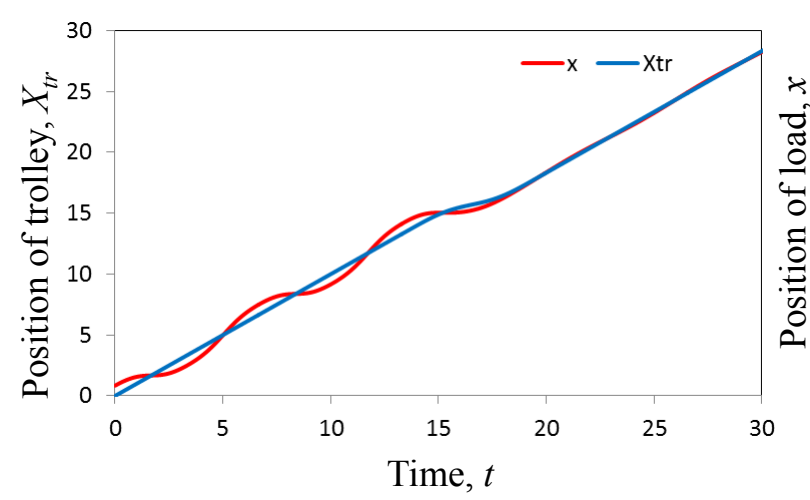

(a)

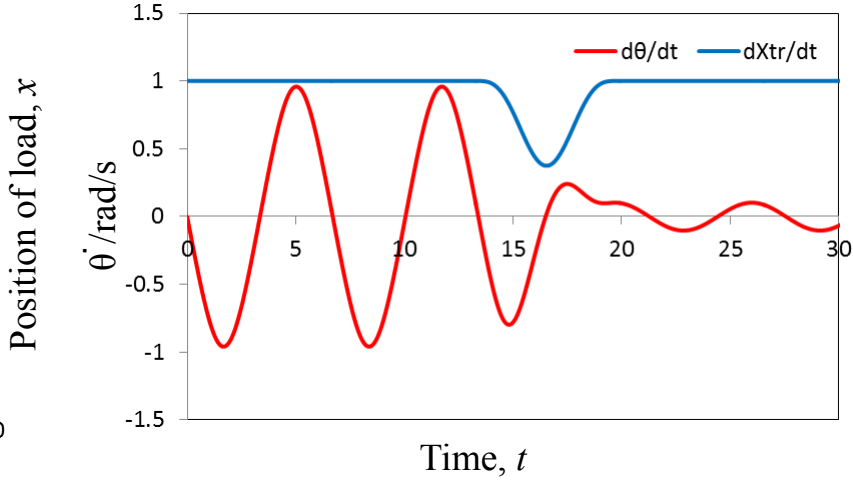

(b)

Fig. 3 Temporal change of (a) the positions of the trolley and the hoisting load or (b) the swing angular velocity and the velocity of the trolley of the crane under the numerical calculation of the suppression of residual vibration with once intermittent enforced displacement during one natural period, in case the initial angle is $1.0 \mathrm{rad}$

After one intermittent feedforward control of VMF in one natural period, larger amount of residual vibration remains with larger initial swing angle of the crane as shown in Fig. 4, where $\eta$ is a damping ratio defined in the following expression, 


$$
\eta=\theta\left(t_{0}+\tau\right) / \theta\left(t_{0}\right)
$$

In this calculation, the parameters of $\alpha_{\mathrm{p}}$ have not affected to the results. Since the overhead travelling crane is a nonlinear oscillator like a pendulum of 1DOF as shown in Eq. (1), it can be approximated to a linear oscillator as shown in Eq. (5) in case of a small swing angle of the wire. On the contrary, in case of a larger amplitude of the swing angle, $\theta\left(t_{0}+t^{\prime}\right)$, the difference between these equations becomes wider. Therefore VMF for the control of a linear oscillator (Kotake, et al., 2014) fails suppressions of residual vibration of the crane. In case of a large swing angle, we can use repeated sampleddata controls to cease residual vibration as shown in the latter section.

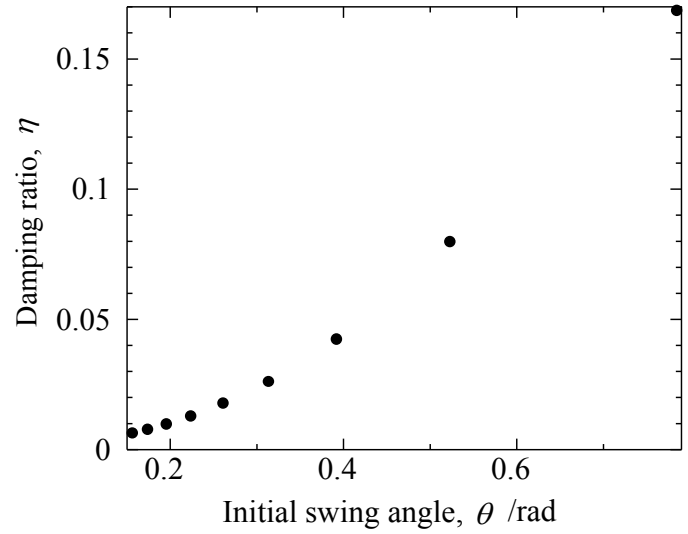

Fig. 4 Relation between damping ratio and initial swing angle of wire in the overhead travelling crane under the suppression of residual vibration with once intermittent enforced displacement for one natural period

\subsection{Robustness of feedforward control to suppress residual vibration}

In this feedforward control procedure, we have to measure the swing angle and swing angular velocity of the wire at the beginning of the operation. In many cases, these values are estimated from acceleration measurements from a sensor in the hoisting load, and some estimation errors can be occurred. Therefore, we have to discuss the robustness of the feedforward operations by using VMF with different estimation errors. In this section, we will mainly discuss the robustness of the proposed feedforward control with error of the initial swing angle and the natural frequency.

At first, we have simulated suppression of residual vibration of the crane under different initial swing angle with some errors. The initial swing angle, which is estimated from data and used for VMF, is called estimated initial swing angle, $\theta_{\text {in,e. }}$ The genuine initial swing angle, which is usually different from estimated one, is called real initial swing angle, $\theta_{\text {in, }}$. As the results of numerical calculations, distribution of the damping ratio is shown in Fig. 5 under different estimated and real initial swing angles. It is sufficient that simulated residual vibration is minimum, when the estimated swing angle is equal to the real one.

Contrary to our expectation, crane oscillation can be well suppressed with $20 \%$ errors of estimation in initial swing angle. So the success of repowering operations by crane experts is the result from the non-sensitive properties in initial swing angle of the repowering manipulation. Moreover, since the perfect suppression trajectory of the trolley, $X_{t r}\left(t_{0}+t^{\prime}\right)$, can be expressed as a linear combination of basis functions of $X_{t r \mathrm{p}}\left(t_{0}+t^{\prime}\right)$, whose subscript $\mathrm{p}$ is an integer, $X_{t r}\left(t_{0}+t^{\prime}\right)$ can be various arbitral forms. Hence, we can conclude that repowering operation does not require so strict technique, by which experts can control the crane with experience.

In second, we have simulated suppression of residual vibration of the crane with some errors in natural frequency. As shown in Fig. 6, ratio between estimated natural frequency for VMF, $\omega$, and genuine one, $\omega_{0}$, sharply affected the damping ratio of the swing oscillation after one feedforward operation. This $\mathrm{v}$-shaped relation between the natural frequency and the damping ratio reminds us the filter-like properties of input/command shaping technique (Singhose, 2003). As shown in Eq. (8) and (11), the virtual enforced base angle of the pendulum and the trajectory of the trolley are the function of $\omega+$ and $\omega$, which are separated with more than half of the natural angular frequencies from $\omega_{0}$. These quantized angular frequencies of VMF could be interrupted as uncertainty principle in intermittent operation with limited interval of natural period like quantum mechanics. 
Since this V-shaped behavior indicates frequency sensitive properties of the proposed method, the natural frequency is one of the most important parameters to be estimated before deciding VMF. Other parameters will also affect the suppression ability of VMF, especially such as the initial swing angular velocity and the position and velocity of the trolley. They will be discussed in another chance.

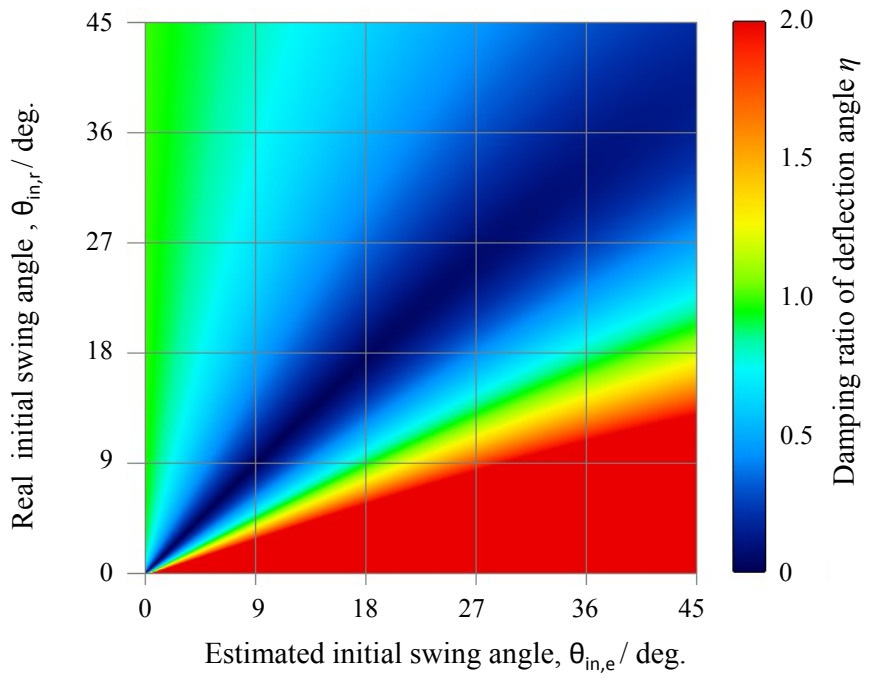

Fig. 5 Damping ratio of the swing angle of the crane after residual vibration suppression with once intermittent enforced displacement at various estimated and real initial swing angles

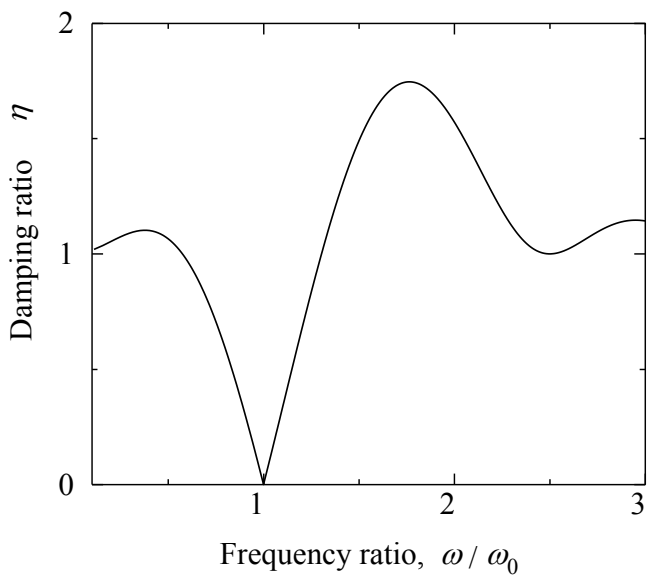

Fig. 6 Relation between frequency ratio with mal-selection and damping ratio of swing oscillation after the suppression of residual vibration with once intermittent enforced displacement by using vibration manipulation function

\section{Sampled-data control to suppress residual vibration under travelling state in inertial system}

\subsection{Basic concept of sampled-data control}

When the hoisting load remains residual vibration in the overhead travelling crane, we can repeat the feedforward control in every natural period by modifying VMF in accordance with the sampled data of the swing angle, $\theta\left(t_{0}+n \tau\right)$, and the swing angular velocity, $\theta\left(t_{0}+\mathrm{n} \tau\right)$, at the beginning of each operation, where $\mathrm{n}$ is the number of repetition. To measure the above quantities, we need to set some sensors to predict them. For instance a sensor of acceleration can be placed at the hoisting load of the crane. Since it takes a certain time to estimate the swing angle and the swing angular velocity in every natural period from the acceleration data of the load, we have to measure them previously by considering certain time lag. To improve the precision, we should measure them more frequently than the interval of the natural period, which is the order of second in a conventional overhead travelling crane. 
To bring the hoisting load to a desired destination, we have to measure the position and velocity of the trolley, which can be estimated from the values of encoder in the motor. Besides measuring them, we also need to measure the acceleration and jerk of the trolley in every natural period. For instance, they can be measured from an acceleration sensor at the trolley. As discussed in the previous section, since the trajectory of the trolley in Eq. (13) is available in inertial flame, the acceleration and jerk of the trolley should be zero at the beginning of the operation to suppress residual vibration well. If the acceleration of the trolley is not zero, there remains some residual vibration after the operation. In many cases it can be suppressed after the repeated operations under the following sampled-data control. Or we should wait for a while to make the travelling speed of the trolley constant.

In inertial flame, the feedforward control can be repeated in every natural period with modifying the function from sampled data. The procedure is known as sampled-data control. Hence the modification of the function is repeated in every natural period, fast PC is not necessary for the feedback process in the sampled-data control. Moreover, this is the first example to define the sampling interval from the physical properties of the controlled system in the field of control theory.

Without external noises and control errors, the system can reduce residual oscillation of the hoisting load as small as possible. However, in practice, since actual overhead travelling crane involves these noises and errors, there are limits of reduction. For instance, under external noises, mechanical energy of the suppressed oscillator cannot be lowered than input energy from the external noise during each operation. Therefore, if the mechanical energy of the hoisting load becomes almost equivalent to that from external noise, repeated operation can be stopped. To prevent the accumulation of oscillation energy from outside, we should restart the operation when the energy of oscillator exceeds certain threshold.

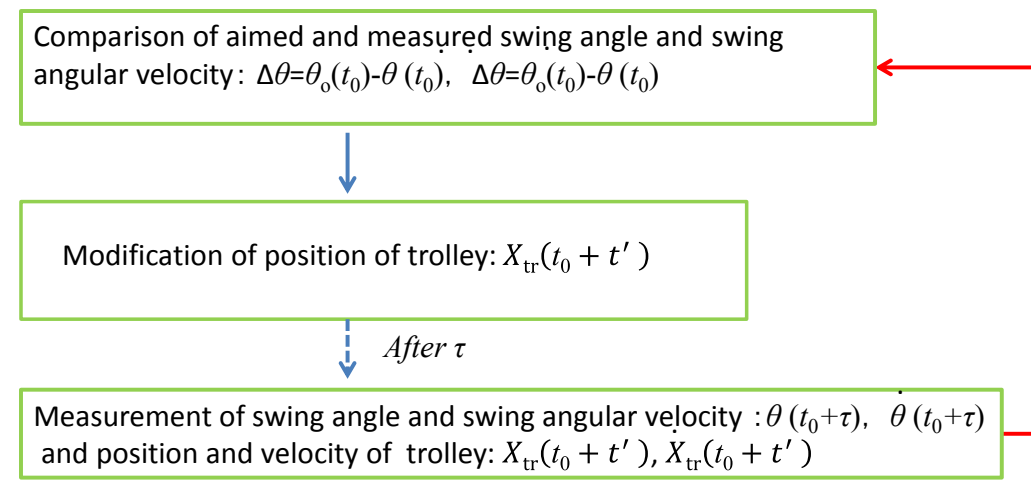

Fig. 7 Flow chart of sampled-data control process for pursuing aimed swing angle and aimed swing angular velocity of the wire by using vibration manipulation function as intermittent feedforward control with periodic feedback modification from sampled data

The flow chart of this sampled-data control is shown in Fig. 7. The unique points of the control are the followings. At first, it is basically feedforward control in the natural period. In second, the sampling time is decided from the property of the 1DOF oscillator as already described. In third, VMF works as lifting function (Yamamoto 1993), which connects digital controller to analog plant in the sampled-data control. In forth, different from former lifting techniques, error deviation is calculated from the difference between discrete sampled data and discrete aimed values. On the other hand, error deviation is calculated from the difference between continuous sampled data and continuous control input in former sampled-data feedback control. At last but not least, the control process with each VMF is equivalent to energy transfer algorithm of virtual three-vibro-impact oscillator under wave circuit described in the control of a linear oscillator (Kotake, et al., 2014).

\subsection{In case operation begins with zero swing angular velocity}

As mentioned in the above section, Eq. (13) is the trajectory of the trolley, when the operation begins with zero swing 
angular velocity, $\dot{\theta}\left(t_{0}\right)=\dot{\theta}$ in $=0$. By using this equation, we simulated the sampled-data control of the overhead travelling crane to suppress residual vibration as shown in Fig. 8(a). In the following calculations, we used $\alpha_{\mathrm{p}}=1$ and $\alpha_{\mathrm{p}}$ ${ }_{\neq 1}=0$ as the parameters. The crane was operated twice to suppress residual vibration of the hoisting load during $t_{01}=2 \pi<$ $t^{\prime}<3 \pi$ and $t_{02}=3 \pi<t^{\prime}<4 \pi$ intermittently during travelling. Comparing the numerical results in Fig.3 (b) and Fig. 8(a), by controlling the crane twice by modifying VMF from sampled data, residual vibration of the crane was disappeared, even in the case of a large initial swing angle. Since the swing angle of the wire can be reduced to be small after the first operation, suppressed vibration can be succeeded with the repeated controls of a linear oscillator. Therefore, robustness of the method can be achieved by the sampled-data control.

\subsection{In case operation begins with non-zero swing angular velocity}

On the other hand, when the operation begins with a non-zero swing angular velocity, $\dot{\theta}\left(t_{0}\right)=\dot{\theta}$ in $\neq 0$, the simulated results are shown in Fig. 8(b). Although residual vibration can also be suppressed quite well after twice intermittent operations of the trolley, the velocity of the trolley has to be changed from the beginning to the end. To recover the travelling speed of the crane, additional operations should be required. Therefore, if people want to keep constant travelling speed of the crane after one operation, he should begin his operation when the swing angular velocity becomes zero.

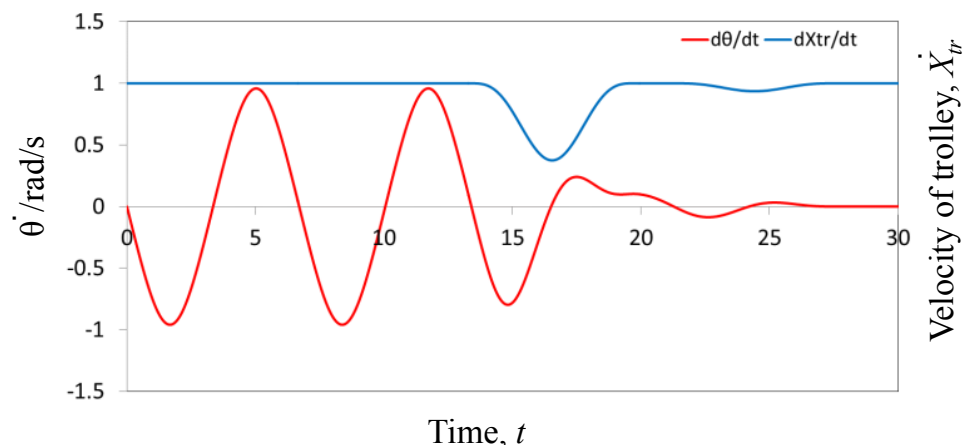

(a)

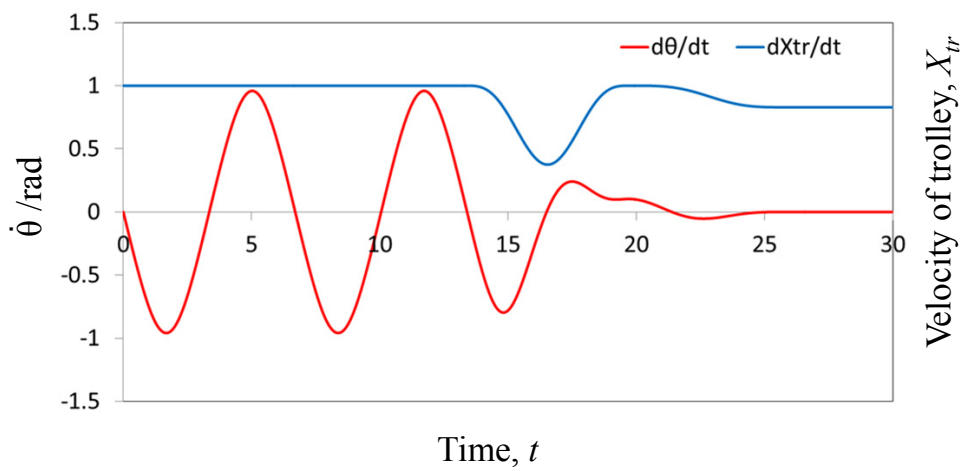

(b)

Fig. 8 Temporal change of the swing angular velocity and the velocity of the trolley of the crane under the numerical calculation of the suppression of residual vibration with twice intermittent enforced displacement during one natural period, in case the initial angle is $1.0 \mathrm{rad}$ and the initial swing angular velocity is (a) $0.0 \mathrm{rad}$ which is started second operation after waiting for this condition, or (b) $0.102 \mathrm{rad}$ which is started second operation just after the first one

\section{Experiment for feedforward control to suppress residual vibration}

\subsection{Experimental conditions}

As shown in the results of numerical calculations, the feedforward control can reduce residual vibration in one natural period. Since the feedforward function is analytically obtained, the crane could be operated by a linear actuator with an inexpensive DC motor or a rather expensive DC linear motor. 
To confirm this system experimentally, the following uniaxial simplified experiment of the overhead travelling crane has been performed. We used a 500mm-long belt-drive linear actuator (TG-47G-SG-30-LC7-KBED, Tsukasa Electric Co. Ltd.) to move its mounting as the trolley. A 40g weight was hung from the mounting of the linear actuator by a 306 $\mathrm{mm}$ long $1 \mathrm{~mm}$ thick string. The natural period of the pendulum was $1.05 \mathrm{~s}$. Since the fixed point of the weight is near its center of gravity, rotational dynamics of the weight was ignored. Through a robber belt, the mounting was driven by a 24V DC gear motor with a magnetic encoder. The reduction ratio of the gear was 30 . Two pulses were signaled from magnetic encoder for one rotation of the motor.

The DC motor was powered from a bipolar DC power supply (BP4610, NF Co. Ltd.), which has $\pm 60 \mathrm{~V}$ voltage or $\pm 10 \mathrm{~A}$ current output for four quadrants with wide band response from DC to $150 \mathrm{kHz}$. Output voltage of the power supply was controlled through a programed waveform from a multifunction generator (WF1974, NF Co. Ltd.), which has wide frequency range from $0.01 \mu \mathrm{Hz}$ to $30 \mathrm{MHz}$ with 2 channel outputs. A sequence function of the waveform was programed on a personal computer.

The dynamics of the suspended weight and the mounting were recorded through video mode $120 \mathrm{fps}$ of a digital camera (Coolpix S8200, Nikon). The temporal change of the position of the actuator was also measured from pulse sequence from the encoder, which was recorded by a digital data logger (midi LOGGER GL220, Graphtec Co. Ltd.).

\subsection{Voltage function of DC motor to manipulate linear actuator}

Output force of the motor $F$ can be deduced from Eq. (2), since the swing angle of the string and the position of the mounting are analytically expressed in Eq. (12) and (11). Hence $M$, which is the sum of the mass of the mounting and the rubber belt and the effective mass of the pulley and the gear, is larger than the mass of the weight, $m$, Eq. (2) can be simplified into

$$
F\left(t_{0}+t^{\prime}\right)=(m+M) \ddot{X}_{t r}\left(t_{0}+t^{\prime}\right)
$$

In case we use a DC motor or a DC linear motor, the following property stands between output force $F$ of the motor and input electric current $i$, electric current of DC motor can be determined as

$$
i\left(t_{0}+t^{\prime}\right)=\left(F\left(t_{0}+t^{\prime}\right)+F_{f}\right) / \kappa_{t}
$$

where $\kappa_{\mathrm{t}}$ is a thrust constant of the motor and $F_{\mathrm{f}}$ is mechanical friction force. Hence the DC motor is consist of electric coil, of which inductance and resistance are $L$ and $R$, applied voltage to the motor can be determined from the following equation analytically.

$$
E\left(t_{0}+t^{\prime}\right)=L \frac{d i\left(t_{0}+t^{\prime}\right)}{d t}+R i\left(t_{0}+t^{\prime}\right)+\kappa_{\mathrm{b}} \eta \dot{X}_{t r}
$$

where $\kappa_{\mathrm{b}}$ is a back electromotive force constant, which is equal to the thrust constant $\kappa_{\mathrm{t}}$, and $\eta$ is a mechanical matching parameter, which is determined from the reduction ratio of the gear and radius of the pulley of the linear actuator. Since the friction force is larger than the inertia of $M$, we ignore the acceleration effect of the linear actuator. Then from Eq. (18), the output voltage becomes

$$
E\left(t_{0}+t^{\prime}\right) \approx \kappa_{\mathrm{b}} \eta \dot{X}_{t r}+E_{0}=\beta \dot{X}_{t r}+E_{0}
$$

From the relation between the velocity of the mounting and the input voltage in static travelling experiment of the linear actuator, we obtain $\beta=58.3 \mathrm{~V} / \mathrm{m}$ and $E_{0}=2.12 \mathrm{~V}$. Correlation coefficient of the experiment is 0.9994 in the single regression analysis.

\subsection{Experimental results to suppress residual vibration}

In the following, we will show the results of the experiment to suppress residual vibration of the weight. The initial swing angle of the string was $0.25 \mathrm{rad}$ and the initial swing angular velocity was $0.0 \mathrm{rad} / \mathrm{s}$. The initial velocity of the 
actuator was $0 \mathrm{~m} / \mathrm{s}$ and the operational period was the natural period of the pendulum, $\tau=1.05 \mathrm{~s}$. In the experiment the programmed output voltage function was determined from Eq. (19) and the time derivative of the trajectory in Eq. (13). From these signals the suspended weight was feedforward controlled. Fig. 9(a) shows the actual position, $X_{t r r e}$, and velocity, $X_{t r}$ re of the linear actuator and the input voltage, $E_{r e}$, for controlling in the experiment and the aimed values of them obtained from numerical calculations. Since all of these experimental data were almost close to the simulated values, the input voltage along Eq. (19) can control the dynamics of the linear actuator. Since magnetic encoder was roughly quantized, measured velocities of the actuator were varied at the maximum speed. That is why the velocity curve of the experiment exhibits bar-like appearance in Fig. 9(a). As shown in Eq. (13) actuator travelled in certain distance during operation.

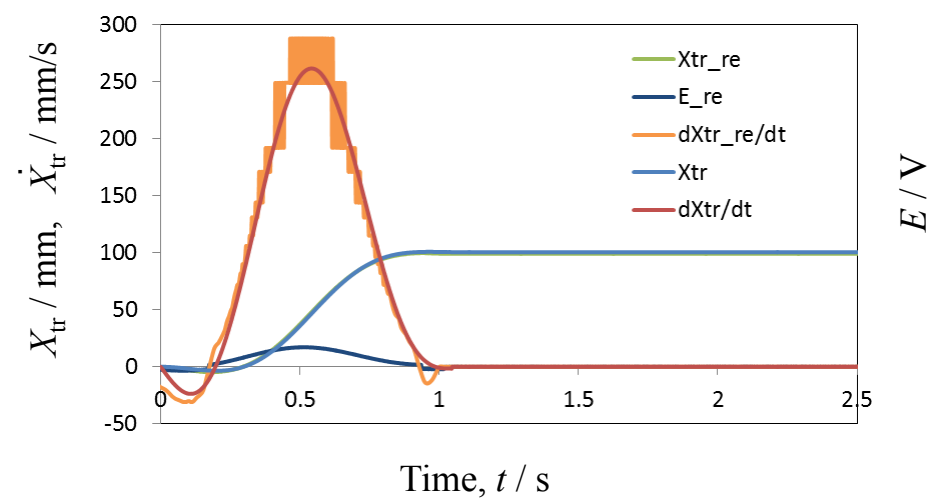

(a)

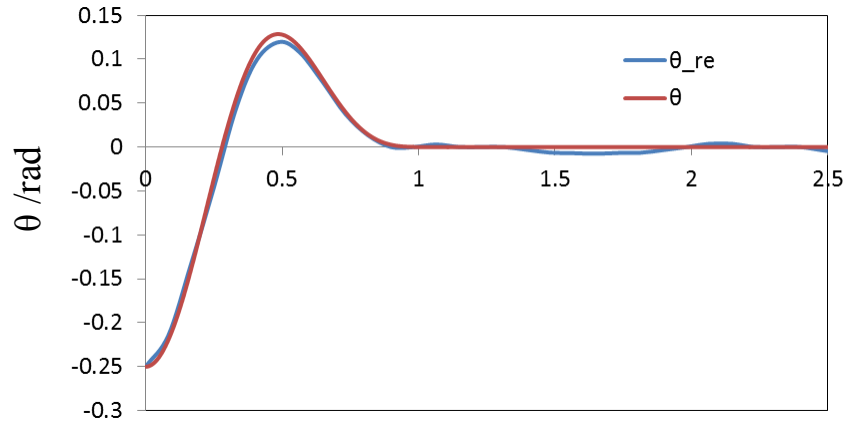

Time, $t / \mathrm{s}$

(b)

Fig. 9 Temporal change of (a) the aimed and observed position and the velocity of the mounting of the linear actuator and applied input voltage or (b) the aimed and observed swing angle of the string in the experiment of the residual-vibration suppression with once intermittent enforced displacement during one natural period, in case the initial angle is $0.25 \mathrm{rad}$ and the initial swing angular velocity is $0.0 \mathrm{rad} / \mathrm{s}$

The aimed swing angle of the string, $\theta$, or measured one, $\theta$ re, during the above feedforward control was shown in Fig. 9(b). Since actual residual vibration was suppressed less than $0.008 \mathrm{rad} \simeq 0.46$ degree after the operation, this feedforward control suppressed residual vibration of the suspended weight but some oscillation remained. Large difference between the theory and the experiment can be observed in the velocity curves of the trolley in Fig. 9(b). Mismatches between them were caused from the effect of inertial force from gears and friction force, which were not precisely concerned in this experiment. Fig. 10 shows twelve stroboscopic pictures of the weight in every $83 \mathrm{~ms}$ interval from right to left. Most left-sided images are overlaid by suppression.

\section{Comparison between sampled-data control with VMF and input/command shaping approach}

Since input/command shaping approach is one of famous methods for feedforward control to suppress residual vibration, it is important and curious to discuss the difference between the shaping approach and the proposed method. 
However, we should mention that the development of VMF is on the way. In this study, the function is limited in certain conditions. At first, the operating period is limited to the natural period of the controlled oscillator, $\tau$, in the proposed method. It is the same in input/command shaping approach. VMF can be developed to be used in an arbitral operating period, $\Delta t$, in the near future.

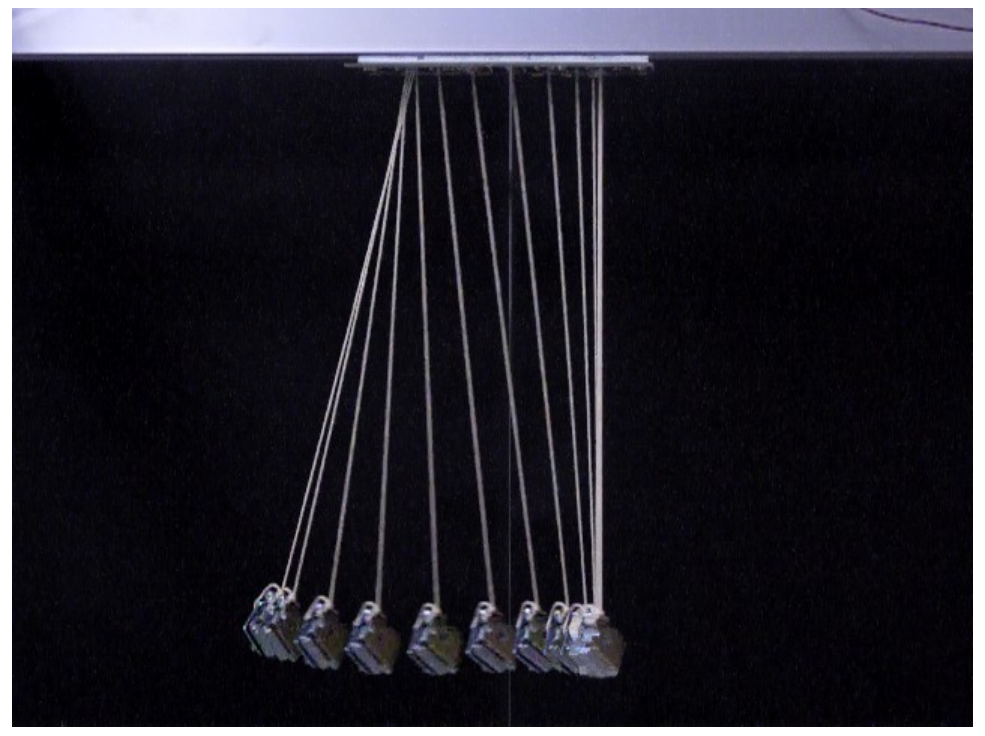

Fig. 10 Stroboscopic pictures of the suspended weight from the mounting of the linear actuator in the experiment of the residual-vibration suppression with once intermittent enforced displacement during one natural period, in case the initial angle is $0.25 \mathrm{rad}$ and the initial swing angular velocity is $0.0 \mathrm{rad} / \mathrm{s}$

In second, VMF is only applicable for a $1 \mathrm{DOF}$ oscillator without damper. The function will also be developed to be used for a 1DOF oscillator with damper, but it takes time. All of these developed conditions can be current disadvantages of the proposed method, since input/command shaping approach is applicable for the 1DOF damped oscillator. This disadvantage will be disappeared after enlarging the conditions.

Besides these weak points, the proposed method has some advantages. At first, it can make closed-loop control to become robust from external noise and internal control error. Since error deviation is evaluated in discrete region in sampled-data control, it can solve the problems of infinite-dimensional model with former lifting functions (Yamamoto, 1994). On the contrary, since input/command shaping approach requires continuous input signal first, it is hard to make closed-loop control in the system (Chang, et al., 2001).

In second, various control input can be deduced from the proposed method, since VMF is a linear combination of infinite basis functions. This variety of the function will be used to choose optimal form from an arbitral evaluation function. However, in shaping approach, since variety of the input control comes from original input function itself, the shaping technique doesn't provide any variety of the input.

In third, discrete aimed values, which are positions and velocities of the mass and the base of the 1DOF oscillator at every sampling period, are merely necessary to determine continuous input function in the proposed method. And other parameters are free to be optimized. On the contrary, people have to decide continuous input function in shaping approach. Although the effect of input function could also be important in shaping technique, not so many discussions have been made.

At last but not least, input/command shaping approach is mainly used to shape input signal to reduce residual vibration. However, since VMF can change aimed position and velocity of the 1DOF oscillator arbitrarily, it can not only reduce residual vibration but can also increase it. By using the proposed method, trajectory of the 1DOF oscillator can be made from series of discrete aimed values.

Input/command shaping technique reduces residual vibration by putting reverse operation in the last half against forward operation at the first half. Since same scenario can be made from the proposed method after enlarging the conditions, it can include shaping technique inside. We will report more relations between the two methods after removing the limits of VMF. 


\section{Conclusions}

In this paper, vibration manipulation function is applied as a feedforward function of enforced acceleration of a trolley to suppress residual vibration of a hoisting load in a one-dimensional overhead travelling crane. The trajectory of the trolley and the swing angle of the wire are obtained as analytical functions in one natural period of the crane. Hence residual vibration can be repeatedly suppressed by sampled-data control in every natural period, weak nonlinear properties of the travelling crane and some errors and external noise are not so large problem to control its oscillation. Numerical calculation confirms the ability of suppression of residual vibration in the crane under travelling state in inertial flame. Moreover, simple experiments were performed to prove the ability of the proposed method. This control method is robust as far as natural frequency of the wire is well estimated and operation timing is appropriate. This analytic feedforward function could be used for an automatic crane machine or a tutoring software for operator's training.

\section{References}

Auernig, J.W. and Troger, H., Time optimal control of overhead cranes with hoisting of the load, Automatica, Vol. 23, No. 4 (1987), pp. 437-447.

Chang, P.-H., Park, J. and Park, J.-Y., Commandless input shaping technique, Proceedings of the IEEE American Control Conference, Vol. 1 (2001), pp. 299-304.

Hindle, T.A. and Singh, T., Robust minimum power/jerk control of maneuvering structures, Journal of Guidance Control and Dynamics, Vol. 24, No. 4 (2001), pp. 816-826.

Kojima, H. and Habiro, K., Optimal trajectory planning of a travelling crane using with genetic algorithm and residual vibration reduction control experiments, Transactions of the Japan Society of Mechanical Engineers, Series C, Vol. 69, No. 682 (2003), pp. 1479-1485. (in Japanese)

Kang, D. and Bien, Z., Design of multiobjective satisfactory fuzzy logic controller using reinforcement learning, International Journal of Fuzzy Systems, Vol. 2, No. 2 (2000), pp. 139-152.

Kotake, S., Yagi, K. and Kawakita, Y., Control method for the trajectory of an oscillator, Japanese Patent Application Publication (2013), Paper No. 2013-185759. (in Japanese)

Kotake, S., Yagi, K. and Kawakita, Y., Control method for the trajectory of an oscillator, International Patent Application Publication (2014), Paper No. PCT/ JP2014/053387. (in Japanese)

Kotake, S., Yagi, K. and Takata, S., Optimal sampled-data vibration manipulation function for one mass-spring system obtained from three vibro-impact oscillators under Grover algorithm, Proceeding of the $12^{\text {th }}$ International Conference on Motion and Vibration Control (2014), Paper No. 2C24.

Manson, G.A., Time-optimal control of and overhead crane model, Optimal Control Applications \& Methods, Vol. 3, No. 2 (1982), pp. 115-120.

Singhose, W., Command shaping for flexible systems: A review of the first 50 years, International Journal of Precision Engineering and Manufacturing, Vol. 10, No. 4 (2009), pp. 153-168.

Solihin, M.I. and Wahyudi, Sensorless anti-swing control for automatic gantry crane system: model-based approach, International Journal of Applied Engineering Research, Vol. 2, No. 1 (2007), pp. 147-161.

Sun, N. and Fang, Y., An efficient online trajectory generating method for underactuated crane system, International Journal of Robust Nonlinear Control, Vol. 24, No. 11 (2014), pp. 1653-1663.

Takata, S., Kotake, S. and Suzuki, Y., Wave algorithm analysis of periodic perfect energy transfer between two globally coupled vibro-impact oscillators (in case of perfect elastic collision at balanced position), Transaction of the Japanese Society of Mechanical Engineers, C, Vol. 77, No. 773 (2011), pp. 14-27. (in Japanese)

Yamamoto, Y., A function space approach to sampled-data system and tracking problems, IEEE Transactions on Automatic Control, Vol. 39, No. 4 (1994), pp. 703-713.

Yi, J., Yubazaki, N. and Hirota, K., Anti-swing and positioning control of overhead traveling crane, Information Sciences, Vol. 155, No. 1-2 (2003), pp. 19-42. 